\title{
One-stage basilic vein tunneled transposition for hemodialysis access
}

\author{
Leszek Sułkowski ${ }^{1}$, Czesław Osuch², Maciej Matyja², Andrzej Matyja²
}

\begin{abstract}
'Department of General Surgery, Regional Specialist Hospital, Częstochowa, Poland ${ }^{2} 2^{\text {nd }}$ Department of General Surgery, Jagiellonian University Medical College, Krakow, Poland
\end{abstract}

Received: 10 June 2020

Accepted: 29 July 2020

Arch Med Sci Civil Dis 2020; 5: e44-e47

DOI: https://doi.org/10.5114/amscd.2020.100838

Copyright @ 2020 Termedia \& Banach

The autogenous arteriovenous fistula (AVF) is an access of choice for chronically hemodialyzed patients [1-4], preferred over central venous catheter placement [5]. The AVF's access vein on the upper extremity can be either the cephalic or basilic vein (BV). The forearm AVF is the first choice. In the case of inadequate, narrow or thrombosed forearm veins, the arm veins can be used to create an AVF. When the favorably located cephalic vein is narrow or thrombosed, the BV remains an alternative [6]. The deep location of BV in the arm makes this vein unsuitable for puncture without superficialization $[1,2,7]$. There are several techniques enabling the use of the deeply located BV, including lipectomy, BV elevation and BV tunnel transposition (BVTT) $[1,2,7,8]$. Therefore, BV AVF remains an access option for difficult cases, along with central venous catheter placement or prosthetic graft AVF [2]. BVTT is technically more complex than catheter placement or prosthetic graft $\operatorname{AVF}[5,9]$, but has all the advantages of autogenous AVF and forms a long and superficial vein segment suitable for cannulation [7].

Surgical technique. A 34-year-old man had been suffering from ESRD and required hemodialysis. Preoperative vein mapping revealed insufficient and too narrow basilic and cephalic veins on the forearm as well as the cephalic vein on the left arm. The diameter of these veins did not exceed $2.5 \mathrm{~mm}$ and mainly was less than $2 \mathrm{~mm}$. The only vein sufficient for AVF creation was the BV on the arm. Due to the deep location of the BV on the arm, the patient was offered a BVTT. The course of the BV on the medial surface of the arm was mapped with ultrasound and marked on the skin (Figure 1, arrows). Under local anesthesia (0.5\% lignocaine) two longitudinal incisions over the BV were performed (Figure 2) and the BV was exposed (arrows) along with its collaterals (arrowheads) and medial antebrachial cutaneous nerve (yellow arrows). The collaterals were ligated to mobilize the BV. The BV was distally dissected at the elbow level and dilated with isotonic saline solution (Figure 3, arrows). The subcutaneous tunnel was formed on the anterior surface of the arm and the BV was transposed into it. Through the distal skin incision, the brachial artery was exposed (Figure 3, arrowheads). End-to-side anastomosis between the brachial artery and the BV was performed with 7-0 suture (Figure 4, arrow). The thrill was palpable on the BV. Finally, the wounds were closed over the suction drain (Figure 5). The drain was removed on the second postoperative day and the patient was discharged

\author{
Corresponding author: \\ Leszek Sułkowski \\ Department \\ of General Surgery \\ Regional Specialist Hospital \\ Phone: +48 792244177 \\ E-mail: leszeksulkowski@ \\ icloud.com
}




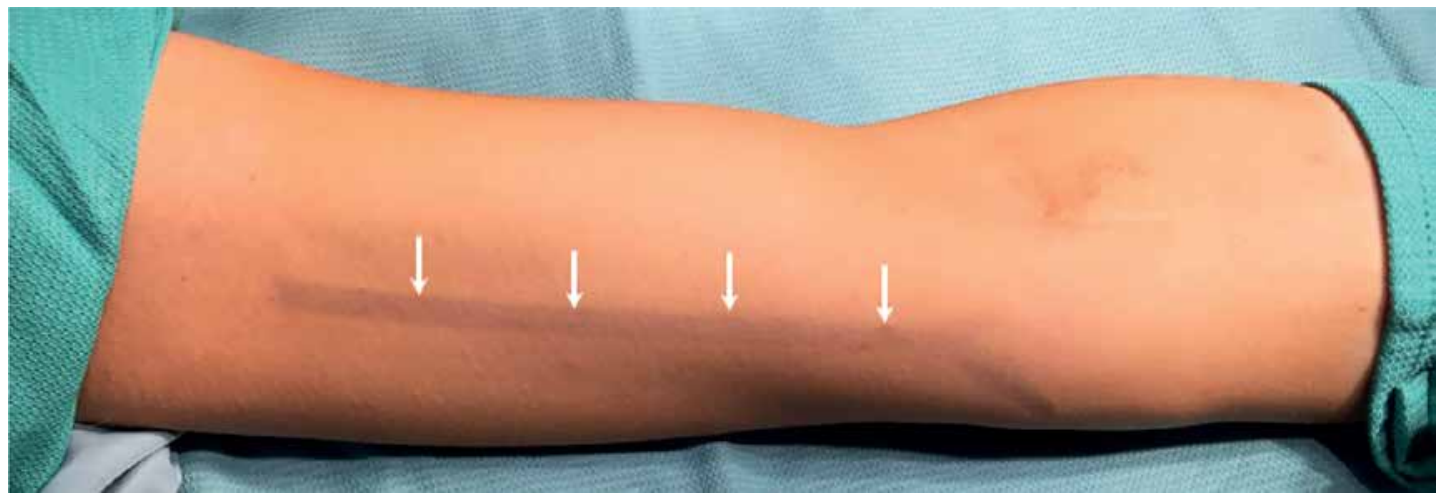

Figure 1. Left arm with basilic vein (arrows) mapped under ultrasound guidance

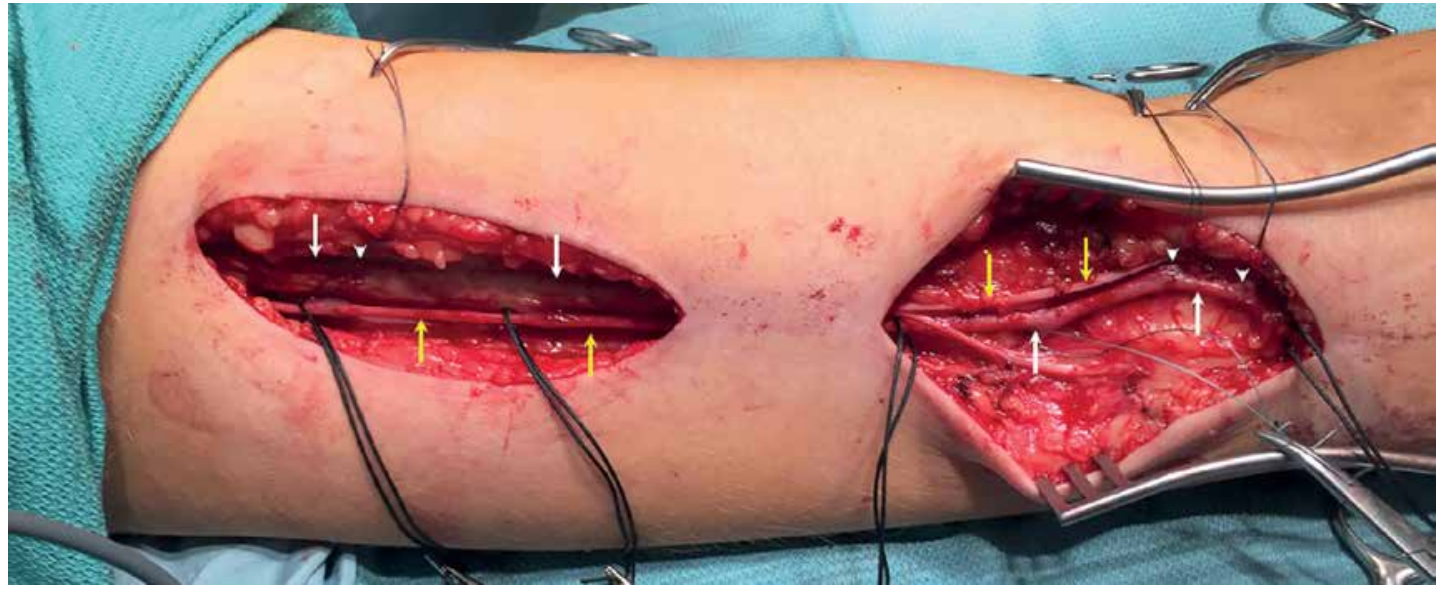

Figure 2. Exposed basilic vein (white arrows), with collaterals (arrowheads) and medial antebrachial cutaneous nerve (yellow arrows)

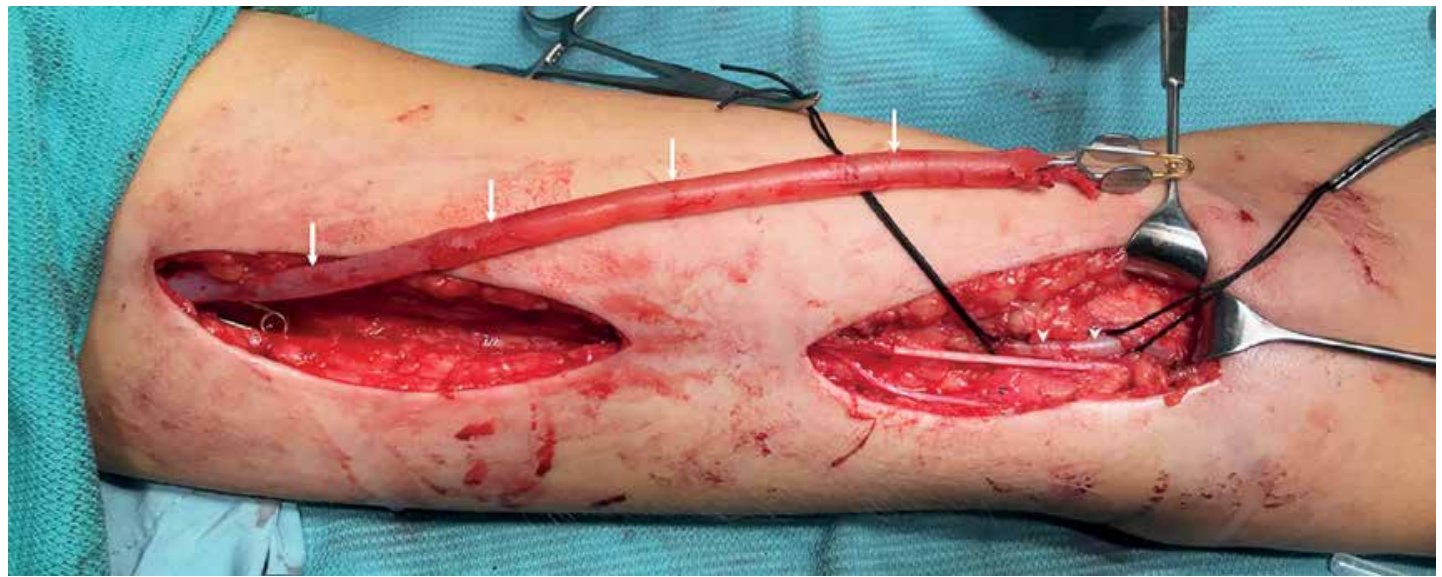

Figure 3. Basilic vein (arrows) with ligated collaterals, dilated with $0.9 \%$ saline solution and exposed brachial artery (arrowheads) in the ulnar fossa

on the $3^{\text {rd }}$ day. The AVF cannulation was attempted 6 weeks later.

Discussion. The cephalic vein compared with the BV is more favorable, superficially located on the anterior surface of the arm and is more frequently used for AVF creation. However, BV AVFs remain an important option for ESRD patients, when the cephalic vein is inadequate for AVF creation, narrow or thrombosed $[1,8,10]$. BV AVFs allow these patients to avoid catheter placement and prosthetic graft AVFs along with complications associated with implantation of catheters or PTFE grafts.

The brachial segment of the BV is deeply and medially located (Figure 2) and therefore it is inadequate for HD without rerouting into subcutaneous tissue, i.e. during the BVTT procedure $[1,8]$. The transposition of the BV subcutaneously may 


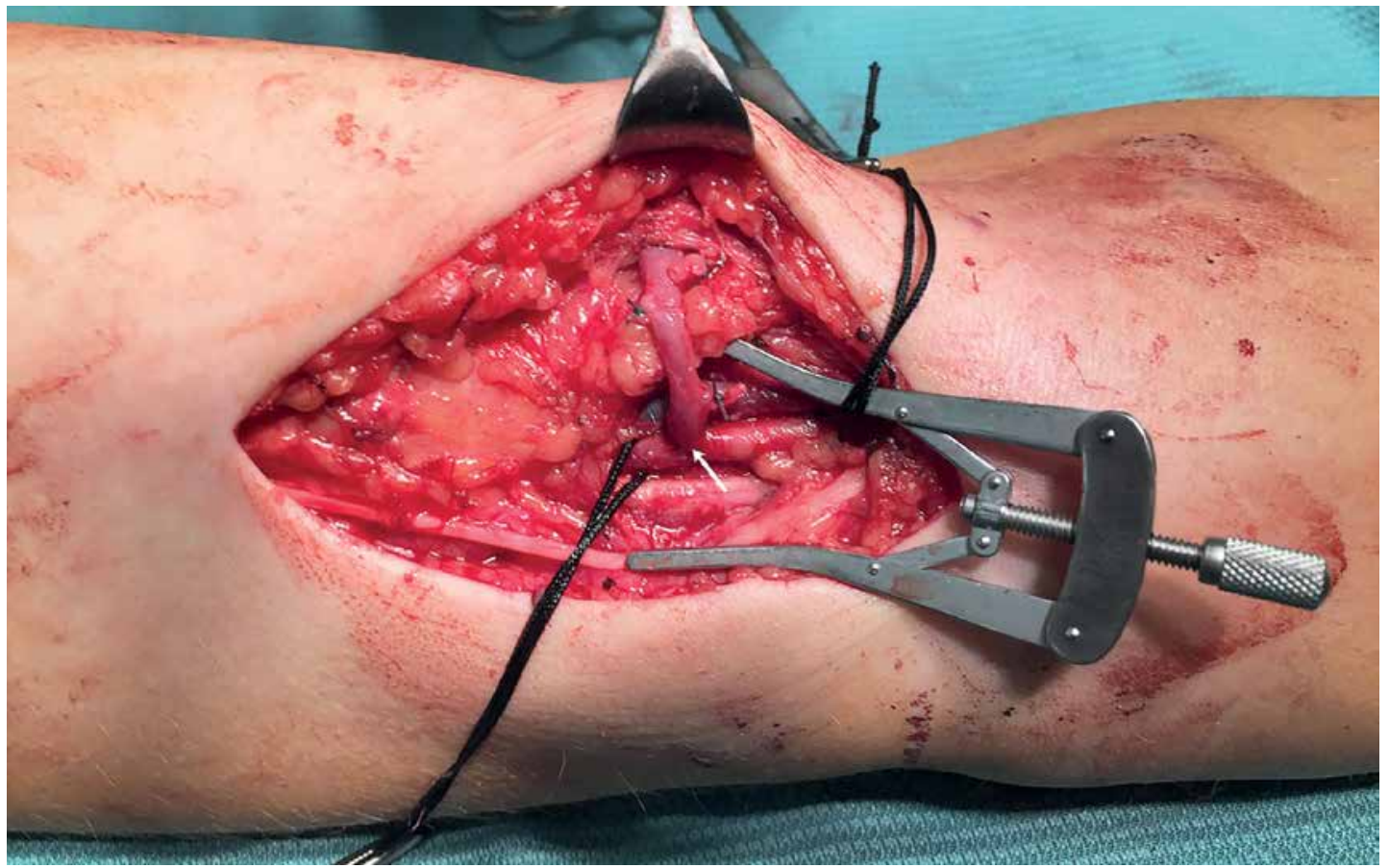

Figure 4. Arteriovenous anastomosis (side of brachial artery - end of basilic vein) (arrow)

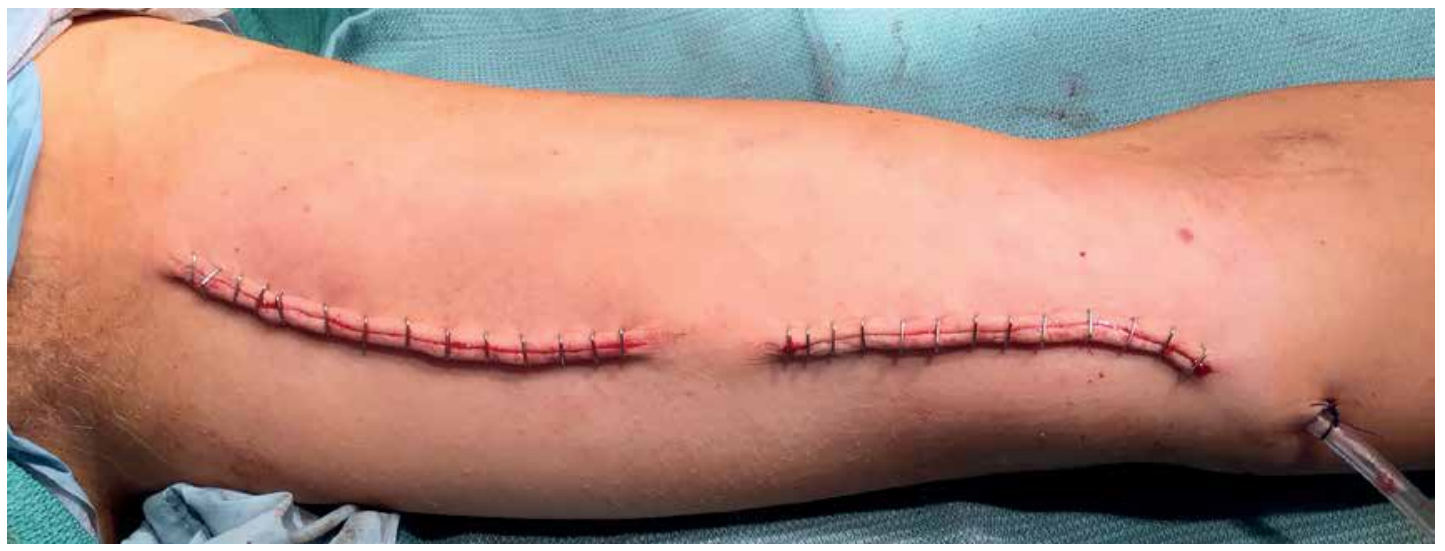

Figure 5. Wounds closed over the suction drainage

be performed during AVF creation (one-stage procedure) or may follow the AVF maturation (twostage procedure, when the first stage is a brachiobasilic arteriovenous anastomosis) [11-14]. The definitive answer to the question of whether one-stage or two-stage technique is optimal is still uncertain and needs a large randomized prospective study. Authors emphasize that maturation, patency, failure and complication rates may suggest equivalence of both techniques. However, it is also emphasized that the strength of the evidence is limited [13, 15-17]. Some authors report that the one-stage technique decreases the time of maturation and the time to the first cannulation. This technique also minimizes the risk of catheter-related infections, since it shortens the time of catheter-based HD [13]. The two-stage procedure is recommended if the basilic vein is less than
$4 \mathrm{~mm}$ in size [15]. We prefer a one-stage BVTT due to the above-mentioned advantages, out of which the short time to the first cannulation and low risk of catheter-related infection are the most important in our opinion.

BVTT requires mobilization of the BV (Figure 3, arrows) and its rerouting into a subcutaneous tunnel on the anterior surface of the arm (Figure 4) to achieve a long, superficial vein segment suitable for cannulation [10]. Pre-operative vein mapping is obligatory. Ultrasound confirms the sufficient diameter of the BV and reveals the BV course, which should be marked on the skin (Figure 1, arrows). Mapping minimizes the intraoperative trauma to both the BV and surrounding tissues (Figure 2).

The surgical approach to the BV may be achieved with a single long incision along the BV or two or more skip incisions [18]. The skip inci- 
sions (Figure 2) allow as wide a view of the BV as a single long incision, but the procedure is more complex and time-consuming and surgery is prolonged. However, skip incisions reduce wound-related complications, i.e. infection or dehiscence, and increase the overall patients' satisfaction with the whole procedure $[18,19]$. We use predominantly two or sometimes three skin incisions along the BV course (Figure 2).

Since the wounds along the arm are long and deep, suction drainage for about two postoperative days is in our opinion mandatory to prevent hematoma formation (Figure 5).

BV transposition is reported to be reliable and durable access also in children, for whom access creation is always challenging due to the small caliber of vessels [6].

Conclusions. Preoperative BV mapping is obligatory. Dividing the skin incision into two or three skip segments makes the BV exposure more difficult, but decreases the rate of wound-related complications. The BV should be transposed to a subcutaneous tunnel. Suction drainage should be routinely used. One-stage BVTT is a complex procedure, but should be recommended for patients with insufficient other veins. It forms a long vein segment suitable for cannulation.

\section{Conflict of interest}

The authors declare no conflict of interest.

\section{References}

1. Sulkowski L, Matyja M, Pasternak A. Lipectomy technique as a second-stage procedure for primarily matured, deep outflow vein in obese individuals. Indian J Nephrol 2018; 28: 320-2.

2. Korkut AK, Kosem M. Superficialization of the basilic vein technique in brachiobasilic arteriovenous fistula: surgical experience of 350 cases during 4 years period. Ann Vasc Surg 2010; 24: 762-7.

3. Sulkowski L, Matyja M, Pasternak A. Management of vascular access steal syndrome after high flow arterio-venous fistula with revision using distal inflow (RUDI) technique. MGM J Med Sci 2018; 5: 129-31.

4. Sulkowski L, Matyja M, Walocha JA, Pasternak A. Management of hemorrhage from dialysis access site: our experience. MGM J Med Sci 2018; 5: 109-11.

5. Sulkowski L, Matyja M, Pasternak A. Salvage technique for complicated hemodialysis patients with central venous occlusion. J Postgrad Med Edu Res 2018; 52: 152-4.

6. Kfoury E, Demaree CJ, Poi MJ, Matos JM, Bechara CF, Lin PH. Long-term outcomes of staged basilic vein transposition for hemodialysis access in children. J Vasc Access 2017; 18: 366-70.

7. Gerrickens MWM, Vaes RHD, Govaert B, Teijink JAW, Scheltinga MR. Basilic vein transposition for unsuitable upper arm hemodialysis needle access segment may attenuate concurrent hand ischemia. Hemodial Int 2018; 22: $335-41$
8. Wang S, Wang MS, Jennings WC. Basilic elevation transposition may improve the clinical outcomes for superficialization of basilic arteriovenous fistula veins. J Vasc Surg 2017; 65: 1104-12.

9. Rego D, Nogueira C, Matos A, et al. Two-stage basilic vein transposition: second stage results. Ther Apher Dial 2018; 22: 73-8

10. Bourquelot P, Karam L, Robert-Ebadi H, Pirozzi N. Transposition, elevation, lipectomy and V-Wing for easy needling. J Vasc Access 2015; 16 Suppl 9: S108-13.

11. Mauro R, Pini R, Bianchini Massoni C, et al. A comparison of two surgical techniques for the second stage of brachiobasilic arteriovenous fistula creation. Artif Organs 2017; 41: 539-44.

12. Przywara S, Ilzecki M, Terlecki P, Zubilewicz T. Axial splitting of the medial antebrachial cutaneous nerve facilitates second-stage elevation of basilic or brachial vein in patients with arteriovenous fistula. J Vasc Surg 2015; 62: 1353-6.

13. Shevitz AJ, Kim AH, Morrow KL, et al. Comparison of patient-specific factors and outcomes for one- and two-stage basilic vein transposition fistulas. J Vasc Surg 2018; 68: 1482-90.

14. Ghaffarian AA, Griffin CL, Kraiss LW, Sarfati MR, Brooke BS. Comparative effectiveness of one-stage versus two-stage basilic vein transposition arteriovenous fistulas. J Vasc Surg 2018; 67: 529-35.

15. Cooper J, Power AH, DeRose G, Forbes TL, Dubois L. Similar failure and patency rates when comparing one- and two-stage basilic vein transposition. J Vasc Surg 2015; 61: 809-16.

16. Tan TW, Siracuse JJ, Brooke BS, et al. Comparison of onestage and two-stage upper arm brachiobasilic arteriovenous fistula in the Vascular Quality Initiative. J Vasc Surg 2019; 69: 1187-95.e2.

17. Sheta M, Hakmei J, London M, et al. One- versus twostage transposed brachiobasilic arteriovenous fistulae: a review of the current state of the art. J Vasc Access 2020; 21: 281-6.

18. Shaikh FA, Nazeer S, Sophie Z, Shahzad N, Siddiqui NA. Multiple skip incisions versus single long incision for single-stage basilic transposition arteriovenous fistula: a cohort study. Ann Vasc Surg 2018; 50: 135-9.

19. Sulkowski L, Matyja M, Walocha JA, Pasternak A. Satisfaction with life among dialyzed patients: a cantril ladder survey. MGM J Med Sci 2018; 5: 6-11. 